\title{
Requisitos Não-Funcionais em Modelos de Processos de Negócio: Uma Revisão Sistemática
}

\author{
Tarcísio C. Pereira ${ }^{1}$, Fernanda M. R. Alencar ${ }^{1}$, Jackson R. F. da Silva ${ }^{1}$, Jaelson F. B. Castro ${ }^{1}$ \\ ${ }^{1}$ Universidade Federal de Pernambuco - UFPE \\ Cidade Universitária, S/N, 50741-000 Recife, Brasil \\ $\{t c p, j r f s, j b c\} @$ cin.ufpe.br, fernanda.ralencar@ufpe.br
}

\begin{abstract}
Adaptability is an important requirement that aims to reconfigure business processes through contextual information. Non-functional requirements (NFRs) are also important for organizations, as they are related to restriction and quality aspects. However, in the business process modeling, NFR and context are hard to be captured and represented. How will be represented the context information and NFR in BPM, if they are? To answer these questions, we conducted a systematic review, the results of which are presented in this article. 1883 papers were initially identified of which only 24 approaches were identified and analyzed.
\end{abstract}

Resumo. Adaptabilidade é um requisito importante que visa a reconfiguração de processos de negócio através de informações contextuais. Requisitos nãofuncionais (RNFs) também são importantes para as organizações, pois estão relacionados a aspectos de restrição e qualidade. Contudo, na modelagem de processos de negócio (do inglês, BPM), contexto e RNF são difíceis de serem capturados e representados. Como, então, são representados informações de contexto e RNF em BPM, se é que o são? Para responder esses questionamentos, foi conduzida uma revisão sistemática, cujos resultados são apresentados neste artigo. Foram identificados inicialmente 1883 trabalhos dos quais apenas 24 propostas foram identificadas e analisadas.

\section{Introdução}

Os processos estão se tornando cada vez mais complexos e heterogêneos, inseridos em ambientes onde as mudanças são constantes, sendo influenciados por fatores geográficos, climáticos, dentre outros. As empresas precisam manter seus processos atualizados e funcionando adequadamente, sem desprezar os requisitos de qualidade. Abordagem dirigida a contexto foi projetada para cobrir essas lacunas através da capacidade de percepção contínua do ambiente do processo e decisões baseadas no controle do processo [Santos et al. 2012]. Porém, as mudanças de contextos para processos de negócio tendem ser dramáticas e as linguagens de modelagens atuais não possuem características de gerenciamento de processos flexíveis [Xia and Wei 2008].

Considerar a qualidade de processos é essencial em futuros sistemas de software [Kedad and Loucopoulos 2011]. As modelagens atuais de processos de negócio capturam atividades que representam aspectos funcionais de um sistema de informação. Enquanto os requisitos ditos de qualidade, restrições ou softgoals, os chamados requisitos não-funcionais (RNF), não são capturados, pois o foco, na modelagem de 
processos de negócio está no comportamento funcional [Wolter and Meinel 2010], [Pavlovski and Zou 2008].

Os RNFs são importantes para as organizações, pois estão relacionados a aspectos de restrição e qualidade, tais como tempo de execução, privilégio de segurança dentre outros. Em [Saeedi et al. 2010], afirma-se que os requisitos de qualidade são o caminho para alcançar performance e satisfação dos clientes e que as modelagens sofrem de alguns problemas: I - a dificuldade de capturar requisitos de qualidade na fase de modelagem; e, II - a impossibilidade de diferenciar serviços que oferecem a mesma funcionalidade. Assim, tem-se como objetivo responder às duas questões de pesquisa seguintes:

- (Q1) Requisitos não-funcionais são representados em BPM?

- Quais são as linguagens de modelagem utilizadas?

- Como os RNFs são representados nas linguagens de modelagens identificadas?

- (Q2) Informações de contexto são consideradas em BPM?

- Como o contexto é representado?

- Como o contexto é capturado?

- Quais são as estratégias de adaptação?

Devido a grande importância desta área de pesquisa, também poderiam ser feitos outros questionamentos, por exemplo, saber se as informações de contexto são consideradas na especificação dos RNFs e identificar respectivas abordagens e problemas existentes. Para este trabalho, foram identificadas abordagens que de fato usam RNFs e contexto em BPM. Utilizando-se da busca às principais bases de dados, foram identificados 1883 trabalhos, dentre os quais foram classificados e analisados 13 trabalhos que levam em conta RNFs na modelagem de processos, 14 que consideram contexto em BPM e 1 trabalho que discute RNF e contexto em BPM.

O restante do trabalho está estruturado da seguinte maneira: na seção 2 é apresentado o processo de revisão sistemática da literatura; o protocolo formal para a revisão sistemática é apresentado na seção 2.1; na seção 3, são descritos e analisados os resultados alcançados; os trabalhos relacionados são discutidos na seção 4; seção 5 aborda as ameaças à validade dos resultados desta pesquisa e, por fim, na seção 6 são apresentados as conclusões e trabalhos futuros.

\section{Revisão Sistemática da Literatura}

A revisão de literatura é o processo central que ampara todo projeto de pesquisa, permitindo que o conhecimento científico seja identificado de forma a possibilitar uma pesquisa planejada, evitando esforços duplicados e repetição de erros anteriores [Dyba et al. 2005].

Uma revisão sistemática (RS), assim como outros tipos de estudo de revisão, é uma forma de pesquisa que utiliza como fonte de dados à literatura sobre determinado tema [Sampaio and Mancini 2007]. Atua como um meio para identificar, avaliar e interpretar toda pesquisa relevante e disponível sobre uma questão de pesquisa específica, tópico ou fenômeno de interesse, utilizando uma metodologia de revisão que seja confiável, rigorosa e que permita auditagem [Mafra and Travassos 2006]. Estabelece um processo formal para conduzir a investigação, evitando a introdução de vieses da revisão de literatura informal, dando maior credibilidade à pesquisa em andamento [Sampaio and Mancini 2007]. A partir da pesquisa num tópico em particular, esse tipo de 
revisão pode induzir à identificação, seleção e produção de evidências, considerando os conhecimentos e as iniciativas existentes no campo de interesse [Mian et al. 2005]). É a partir dessas evidências que as perguntas de pesquisa serão respondidas.

A revisão sistemática envolve um conjunto de atividades, segundo um protocolo, que guiará todo o processo de condução da revisão. As três fases do processo de condução são: planejamento da revisão, execução da revisão e análise e divulgação dos resultados. Na Figura 1, têm-se as fases do processo de revisão sistemática propostas em [J. H. Little and Pillai 2008], [Mafra and Travassos 2006] e [J. Biolchini 2005]. Essas fases não são necessariamente sequenciais, pois possuem iterações, fazendo com que muitas atividades sejam iniciadas na fase de planejamento e refinadas posteriormente.

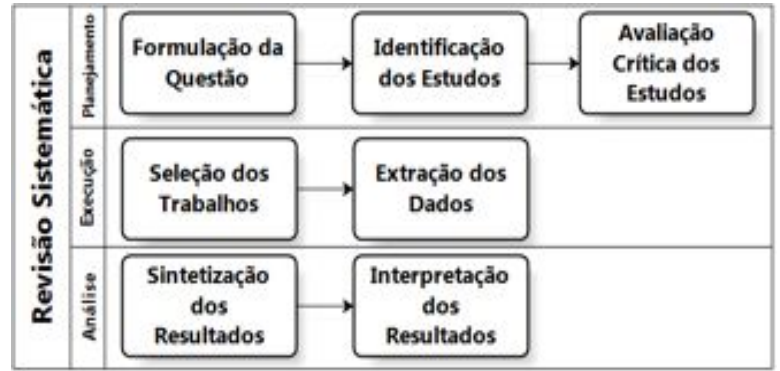

Figura 1. Processo de Condução da Revisão Sistemática

\subsection{Condução da Pesquisa}

As strings de busca foram definidas de acordo com as perguntas de pesquisa e artigos relevantes da área. A lista completa das strings de busca e suas combinações são apresentadas na Tabela 1. Essas strings foram aplicadas de acordo com o formato exigido por cada engine de busca utilizada (IEEE Xplore, Scopus e Engineering Village), podendo sofrer alterações para que o mecanismo consiga executá-las. Como resultado da aplicação da estratégia de busca, 2252 estudos foram coletados. Após excluir os repetidos, restaram 1883 estudos.

Tabela 1. Strings de pesquisa do protocolo de busca da RS

\begin{tabular}{lc}
\hline Strings & Questões \\
\hline (1) (non-functional requirements OR NFR OR quality requirements OR quality attributes) & Q1 \\
AND (business process OR business process modeling OR BPM) & \\
\hline (2) (context-aware) AND (business process OR business process modeling OR BPM) & Q2 \\
\hline
\end{tabular}

Neste ponto, os artigos foram excluídos de acordo com os critérios de exclusão:

- Estudos que não discutem (contexto e requisitos não-funcionais) ou (requisitos não-funcionais e modelagem de processos de negócio) ou (contexto e modelagem de processos de negócio) ou (contexto, requisitos não-funcionais e modelagem de processos de negócio);

- Trabalhos que apresentem resultados sem esclarecer os métodos utilizados;

- Estudos disponíveis apenas como resumos;

- Trabalhos incompletos; e

- Estudos duplicados. Quando um estudo foi publicado mais de uma vez, a versão mais completa será considerada. 


\subsection{Seleção dos Estudos}

Primeiramente, os critérios de exclusão foram aplicados no título, procurando identificar as palavras-chave e analisar quais estavam disponíveis por completo, resultando em 133 estudos. Em seguida, uma segunda seleção foi realizada analisando o abstract dos estudos. Ao fim desta etapa, restaram 61 estudos. Um último filtro ainda foi realizado, analisando a introdução e conclusão, que resultou ao final em 24 estudos. A Figura 3 exibe a quantidade de estudos referentes às perguntas de pesquisa dividos por tema e a Figura 3 refere-se à quantidade de trabalhos identificados por ano.

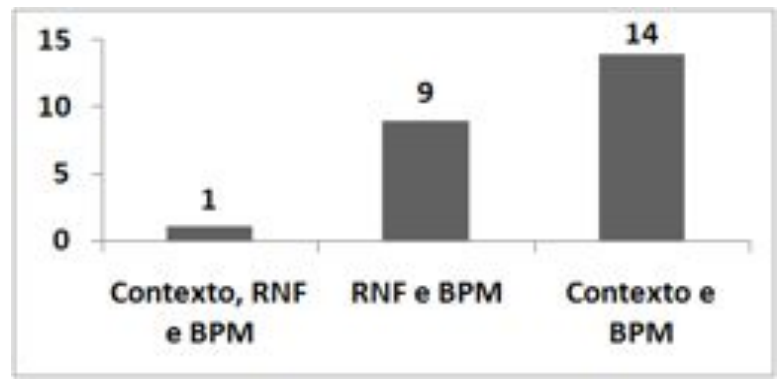

Figura 2. Quantidade de Trabalhos por Tema

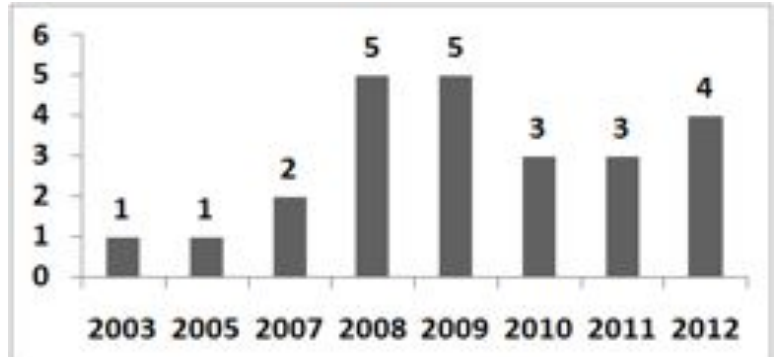

Figura 3. Quantidade de Trabalhos Relevantes por Ano

\subsection{Extração dos Dados}

As informações foram extraídas e armazenadas em formulários padronizados para catalogação e avaliação posterior desses dados para alcançar os objetivos desse estudo. As informações que são armazenadas nestes formulários são as seguintes: título; ano; argumentação central (problema da pesquisa e a solução proposta); as possíveis respostas para as questões de pesquisa; e, referências relevantes.

\section{Análise dos Resultados}

Nesta seção, é apresentado os resultados para as questões de pesquisa, destacando evidências recolhidas a partir dos formulários de extração de dados. Além dos 24 trabalhos, foram identificados outros 3 potencialmente relevantes à pesquisa que não haviam sido identificados na busca às bases de dados. Ao todo, 27 trabalhos foram identificados. Não consideramos analisar a qualidade das propostas. Um possível indicador de qualidade é o índice de citações.

\subsection{Q1 - Requisitos não-funcionais são representados na modelagem de processos de negócio?}

Para organizar os trabalhos selecionados para responder esta pergunta de pesquisa, criamos uma classificação a partir de uma leitura criteriosa dos estudos. Através da leitura 
de cada trabalho, identificou-se a linguagem de modelagem de processos que foi utilizada para representar os RNFs e então uma tabela foi criada com este mapeamento. A Tabela 2 exibe a classificação de todos os trabalhos identificados, os que estão com um asterisco* ao lado significa que foram inseridos manualmente. Por restrições de espaço, não foi possível entrar em alto nível de detalhe dos trabalhos classificados na Tabela 2. Os parágrafos seguintes descrevem rapidamente como os RNFs são representados na modelagem de processos.

Tabela 2. Classificação dos trabalhos analisados da RS

\begin{tabular}{ll}
\hline \multicolumn{1}{c}{ Classificação } & \multicolumn{1}{c}{ Trabalhos } \\
\hline \hline $\begin{array}{l}\text { Extensão da notação BPMN para representar } \\
R N F\end{array}$ & [Bartolini et al. 2012] \\
& [Bocciarelli and D'Ambrogio 2011] \\
& [Pavlovski and Zou 2008] \\
& [Saeedi et al. 2010] \\
& [Santos et al. 2012] \\
& [Serrano et al. 2009]* \\
& [Wolter and Meinel 2010] \\
& [Xavier et al. 2009]* \\
\hline Diagrama de Atividades para representar RNF & [Aburub et al. 2007] \\
& [Khaluf et al. 2011]* \\
\hline $\begin{array}{l}\text { Extensão de notação Stock and Flow Diagrams of System Dynamics } \\
\text { para representar RNF }\end{array}$ & [Kedad and Loucopoulos 2011] \\
\hline Modelagem de processos de negócio orientada & \\
a objetivos & [Cardoso et al. 2009] \\
\hline
\end{tabular}

[Bartolini et al. 2012] cria uma abordagem chamada Q4BPMN (Quality for $B P M N$ ), que é uma extensão da notação BPMN, no qual o modelo é anotado com requisitos de qualidade. Um analista identifica os RNFs através da análise de um modelo mapeado em BPMN e então, através de um conjunto de passos, os RNFs são mapeados para uma tarefa, um participante da tarefa ou para toda a "coreografia".

[Bocciarelli and D'Ambrogio 2011] estende a notação BPMN criando uma abordagem chamada PyBPMN (Performability-enabled BPMN) que lida com aspectos nãofuncionais. Este trabalho emprega a caracterização de performance e confiança em processos de negócio. A extensão proposta é baseada na abordagem que explora princípios e padrões da MDA (Model Driven Architecture), a representação gráfica é feita a partir da associação entre um RNF e uma atividade, por exemplo, se a restrição tempo de execução $\leq 2500 \mathrm{~ms}$ está associada a atividade "X", então a mesma tem que ser completada em não mais que 2.5 segundos.

[Pavlovski and Zou 2008] propõe dois novos artefatos que são a operational condition e o control case como uma extensão para a notação BPMN para modelar requisitos não-funcionais. A operational condition é responsável por identificar restrições como performance de uma tarefa, políticas de segurança ou disponibilidade e associá-las a uma atividade, ou seja, é um artefato que está associado a uma atividade de negócio. O control case captura os riscos de negócio associado a uma operational condition e controla os negócios a serem postos em prática para mitigar o risco.

[Saeedi et al. 2010] estende a notação BPMN para apoiar os requisitos de qualidade. Os alvos da extensão são os elementos de atividades do BPMN. Dentro das atividades pode conter a letra "Q"no canto superior direito, que se expandida aparece uma lista dos atributos de qualidade correspondente aquela atividade. Cada RNF dentro de uma atividade pode receber um valor, através desses valores, o autor propõe a utilização de regras de redução para avaliar o modelo. Regras de redução são utilizadas repetidamente em um processo de negócio até restar uma tarefa, esta tarefa contém métricas de requisitos 
de qualidade correspondente a todo o processo de negócio.

[Santos et al. 2012] propõe uma abordagem que utiliza requisitos não-funcionais associados a contexto com o propósito de configurar processos de negócio. Como o objetivo deste trabalho está relacionado com RNFs, será considerado apenas sua utilização dentro da abordagem proposta. Uma vez definido os RNFs, os mesmos são ligados às variantes do processo, essas ligações são representadas através de matriz, que segundo o autor é uma solução escalável para representar este tipo de informação. Para efetuar as ligações, o autor utiliza uma escala qualitativa do NFR Framework criado por [Chung et al. 2000], que consiste de um guia de requisitos não-funcionais, tais como segurança, desempenho e custo, para direcionar o mapeamento dessas restrições em processos.

[Serrano et al. 2009] estende a notação BPMN através da utilização de IP-MAP (Information Products Maps). IP-MAP permite a especificação de processos por meio de um mapa conceitual, no qual as atividades que estão relacionadas à gestão de qualidade de dados são tratadas. Então, a notação BPMN foi estendida para apoiar a qualidade de dados oferecida pelo IP-MAP, com o objetivo de conseguir obter uma notação rica. O resultado consiste na adição de um símbolo no formato de estrela para representar qualidade de dados controlada pelo processo.

[Wolter and Meinel 2010] estende a notação BPMN para englobar requisitos de segurança como confidencialidade, integridade, autenticação e autorização. O autor cria atributos de decoração, que são símbolos, para representar os requisitos de segurança e os associa a cada elemento do modelo. Os atributos podem ser estendidos baseado em papéis, atividades realizadas por humanos e raias.

[Xavier et al. 2009] cria a abordagem BPMNFR com o intuito de integrar a notação BPMN e RNFs, descritos no NFR Framework. Uma letra referente ao RNF é inserida dentro do modelo para que o BPMN seja capaz de representar os RNFs, e um conjunto de passos são descritos para guiar a descoberta dos RNFs a partir de um modelo gerado em BPMN e também a escolha de qual RNF deverá ser inserido no modelo. O trabalho também estende o NFR Framework com o requisito de qualidade usabilidade.

[Aburub et al. 2007] propõe uma abordagem para aplicar o conceito de RNF na modelagem de processos desenvolvendo uma extensão para a notação RAD (Role Activity Diagram), utilizada para mapear aspectos funcionais de processos. Através de um conjunto de passos, os RNF são elicitados e mapeados para o modelo representado pelo RAD. Operacionalizações também são descritas, assim como quais atores são responsáveis por alcançar a melhoria do processo.

[Khaluf et al. 2011] expõe uma nova maneira de modelar restrições de qualidade em processos. $\mathrm{Na}$ abordagem proposta, os processos são modelados em Diagramas de Atividade da UML 2.0, e as restrições de qualidade representadas por PPSL (Process Pattern Language Specification). O diagrama gerado recebe dois novos símbolos, um para indicar se na atividade do processe existe ou não restrições de qualidade, e outra para indicar quais restrições estão presentes em uma atividade.

[Kedad and Loucopoulos 2011] propõe um Framework que oferece suporte para a avaliação de qualidade de processos de negócio através de um modelo cuja notação é a Stock and Flow Diagrams of System Dynamics. Uma das fases desse framework chama-se Enrichment of the BP Metamodel, dado um modelo de processo, o mesmo 
é enriquecido com todas as propriedades requeridas para descrever vários aspectos de qualidade relacionado ao processo de negócio. O resultado é um modelo que descreve elementos do processo e qualidade do processo. Os atributos de qualidade podem ser inseridos dentro das atividades ou do processo inteiro, e a representação desses atributos se dá através do nome do RNF dentro de uma atividade ou através de ligações entre atividades.

[Cardoso et al. 2009] cria uma maneira sistemática de elicitar objetivos a partir de uma configuração organizacional. O trabalho utiliza a linguagem de modelagem Tropos, que foi derivada do NFR Framework para a modelagem de processos. Através de um catálogo de RNFs, os objetivos do negócio são elicitados assim como suas operacionalizações. Na linguagem de modelagem Tropos, os RNFs são representados graficamente através de nuvens. [Lapouchnian et al. 2007] propõe uma abordagem de configuração de processos de negócio através de RNF baseada no NFR Framework, no qual os RNFs são representados graficamente através de nuvens com nome do RNF dentro da mesma. Então, através de um conjunto de passos, o processo de negócio é projetado e enriquecido com novas notações até chegar no último passo onde a configuração do processo é realizada baseada em um determinado RNF.

Após análise dos trabalhos que foram identificados na revisão sistemática e aqueles inclusos manualmente, conclui-se que "sim", os requisitos não-funcionais estão sendo representados na modelagem de processos de negócio. Convencionalmente as modelagens de processos cobrem características funcionais do processo, enquanto que os RNFs são atributos de qualidade que geralmente não são capturados ou identificados. Para cobrir esta lacuna, pesquisadores estão propondo novas abordagens, estendo abordagens já existentes e também encontrando diferentes maneiras de representar os RNFs em processos. A Tabela 3 exibe um mapeamento entre os tipos de representação de RNFs e seus determinados autores.

Tabela 3. Relação entre o tipo de representação de RNF e trabalhos selecionado

\begin{tabular}{|c|c|c|c|c|c|c|c|c|c|c|c|c|c|}
\hline Tipo de Representação - Trabalhos Selecionados & 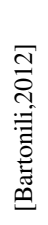 & 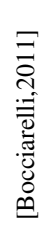 & 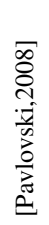 & 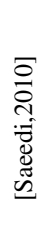 & $\begin{array}{l}\bar{N} \\
\tilde{O} \\
\tilde{N} \\
\tilde{0} \\
\stackrel{0}{0} \\
\tilde{\tilde{D}}\end{array}$ & 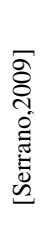 & 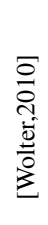 & 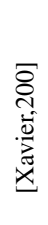 & 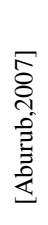 & 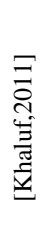 & 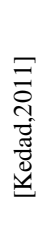 & $\begin{array}{l}\bar{\delta} \\
\delta \\
i \\
\delta \\
\delta \\
o \\
o \\
0 \\
0 \\
0\end{array}$ & 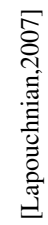 \\
\hline RNF anotado textualmente nos elementos do modelo & $\bullet$ & & & $\bullet$ & & & & $\bullet$ & & & $\bullet$ & & \\
\hline Associação textual entre RNF e elementos do modelo & & $\bullet$ & & & & & & & $\bullet$ & & & & \\
\hline Extensão da notação BPMN com criação de artefatos & & & $\bullet$ & & & & & & & & & & \\
\hline Representação externa do RNF ao modelo de negócio & & & & & $\bullet$ & & & & & & & & \\
\hline Criação de símbolos para representar os RNFs & & & & & & $\bullet$ & $\bullet$ & & & $\bullet$ & & & \\
\hline NFR Framework ou derivados & & & & & & & & & & & & $\bullet$ & $\bullet$ \\
\hline
\end{tabular}

Analisando a Tabela 3 percebe-se que o maior tipo de representação de RNF se dá anotando elementos de um modelo com o nome do RNF, 4 dos 13 trabalhos adotam este tipo de representação. Em seguida, 3 trabalhos representam os RNFs através da criação de símbolos para representá-los. Um empate ocorre entre aqueles que fazem associação textual entre RNF e elementos do modelo e os que utilizam o NFR Framework para representar RNF, cada um com 2 trabalhos. Por fim, encontra-se 1 trabalho que propõe a criação de novos artefatos e um outro que representa os RNFs externamente ao modelo de negócio. 


\subsection{Q2 - Informações de contexto são consideradas na modelagem de processos de negócio?}

Para responder esta pergunta de pesquisa, uma leitura criteriosa dos estudos foi realizada. A partir da análise de cada trabalho, foi possível identificar itens mais gerais, por exemplo, se o resultado do trabalho é uma abordagem ou framework, se descreve alguma ferramenta para apoiar o processo criado, se testes foram realizados com o intuito de validar a proposta e até mesmo se os trabalhos discutem algumas estratégias de adaptação de processos de negócio.

Itens característicos de cada proposta também foram identificados, por exemplo, o tipo de método utilizado para representar as informações contextuais e também a técnica usada para capturar o contexto do ambiente em que o processo de negócio está inserido. A Tabela 4 apresenta uma sumarização dos resultados alcançados através da análise dos trabalhos que tratam de informações contextuais em processos de negócio. Por questão de espaço, não será possível entrar em níveis mais detalhados sobre os trabalhos selecionados, assim como, não foi possível incluir todas as referências da Tabela 4 neste trabalho. Para resolver este problema, as referências estão disponíveis em um website ${ }^{1}$.

Tabela 4. Sumarização dos Resultados

\begin{tabular}{|c|c|c|c|c|c|c|c|c|c|c|c|c|c|c|}
\hline & 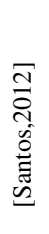 & 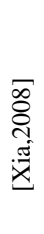 & 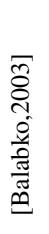 & 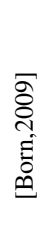 & 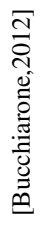 & 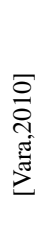 & 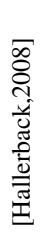 & 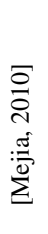 & 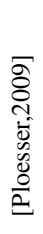 & 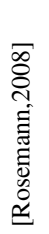 & 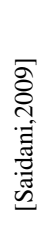 & 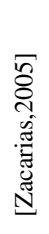 & 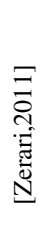 & 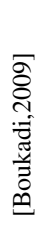 \\
\hline \multicolumn{15}{|l|}{ Itens Gerais } \\
\hline Tipo de Contribuição - Abordagem & $\bullet$ & $\bullet$ & & & & $\bullet$ & $\bullet$ & $\bullet$ & & & $\bullet$ & $\bullet$ & & \\
\hline Tipo de Contribuição - Framework & & & $\bullet$ & $\bullet$ & $\bullet$ & & & & $\bullet$ & $\bullet$ & & & $\bullet$ & $\bullet$ \\
\hline Ferramentas de Suporte & & $\bullet$ & & & $\bullet$ & & & & & & & & & \\
\hline Testes da Abordagem/Framework & $\bullet$ & & $\bullet$ & & $\bullet$ & $\bullet$ & & $\bullet$ & & $\bullet$ & & $\bullet$ & $\bullet$ & $\bullet$ \\
\hline Discute Estratégias de Adaptação & & $\bullet$ & & & $\bullet$ & & & & $\bullet$ & & & & & \\
\hline \multicolumn{15}{|l|}{ Método de Representar Contexto } \\
\hline $\begin{array}{l}\text { Fórmulas } \\
\end{array}$ & & & & $\bullet$ & & $\bullet$ & & & & & & & & \\
\hline Regras & $\bullet$ & $\bullet$ & & & & & $\bullet$ & $\bullet$ & & & & & $\bullet$ & \\
\hline Combinação de Propriedades & & & & & $\bullet$ & & & & & & & $\bullet$ & & $\bullet$ \\
\hline Modelo Gráfico & & & $\bullet$ & & & & & & & $\bullet$ & $\bullet$ & & & \\
\hline Descrição Textual & & & & & & & & & $\bullet$ & & & & & \\
\hline \multicolumn{15}{|l|}{ Técnica de Captura de Contexto } \\
\hline Monitor & $\bullet$ & $\bullet$ & & & $\bullet$ & & $\bullet$ & & & & & & & \\
\hline Mineração de Processos & & & & & & & & & & & & & $\bullet$ & \\
\hline Taxonomia de Informação Contextual & & & & & & & & & & & & & & $\bullet$ \\
\hline Não Especificam ou Não deixam Claro & & & $\bullet$ & $\bullet$ & & $\bullet$ & & $\bullet$ & $\bullet$ & $\bullet$ & $\bullet$ & $\bullet$ & & \\
\hline
\end{tabular}

A Tabela 4 exibe os diferentes tipos de representação de contexto e também as técnicas de captura de contexto que são utilizadas pelas abordagens. Contudo, não deixa claro quais são as estratégias de adaptação utilizadas. Ao todo, foram identificados 11 estratégias de adaptação. As 5 seguintes são definidas por [Xia, 2008]:

- Alternative Task Strategy: Quando uma tarefa falhar, uma tarefa alternativa continua a execução do processo;

- Load Distributed Strategy: Caminhos adicionais são úteis para reduzir o carregamento de recursos;

- Redundant Strategy: Tarefas executadas em paralelo para alcançar determinada atividade de negócio;

${ }^{1}$ www.cin.ufpe.br/ tcp 
- Incremental Strategy: Repetir a tarefa utilizando um mesmo recurso;

- Algorithm for Adaptation: Algoritmo que incorpora diferentes mudanças e estratégias de adaptação dentro do ambiente de negócio.

As estratégias de Re-Refinement, onde o mecanismo de refinamento tem como objetivo compor automaticamente fragmentos de processos disponíveis levando em consideração o objetivo de uma atividade e a configuração de contexto atual e Backward Adaptation que visa trazer de volta uma instância do processo, são discutidas por [Bucchiarone, 2012]. Em seu estudo, [Ploesser, 2009] discute 4 estratégias de adaptação:

- Incremental Learning: A organização incrementalmente expande seu repertório de respostas a situações excepcionais;

- Strategy Change: Quando as organizações enfrentam mudanças de longa duração e previsíveis que podem explorar o repertório clássico de técnicas de reengenharia de processos;

- Contingency Planning: É necessário considerar técnicas de planejamento de contingência quando organizações se preparam para mudanças repentinas e perturbadoras que podem ser difíceis de prever;

- Context-awareness: Organizações em oscilação, expostas à mudanças frequentes e previsíveis, devem considerar contexto como uma estratégia no projeto de processos.

Após a análise dos trabalhos, conclui-se que "sim", informações de contexto estão sendo consideradas na modelagem de processos de negócio. Devido as empresas estarem inseridas em ambientes onde mudanças são constantes, elas precisam manter seus processos atualizados e funcionando adequadamente. Pesquisadores estão propondo ou adaptando abordagens de processos de negócio já existentes para incluir informações para representar os diferentes tipos de contexto. Assim, devido ao reconhecimento da mudança do ambiente, os processos podem se adaptar seguindo alguma estratégia, e continuar funcionando perfeitamente nas condições que foram impostas.

\section{Trabalhos Relacionados}

Em [Salles and Fantinato 2012] foi feito um estudo similar ao nosso, no qual os autores apresentam uma análise sistemática visando buscar trabalhos sobre métodos e técnicas utilizados para associar RNFs a modelagem de processos de negócio. A busca realizada pelos autores também capturou trabalhos que associam outras características de qualidade a processos de negócio, como SLA e QoS (do inglês: Service Level Agreement e Quality of Service respectivamente). Os autores limitaram a busca à um intervalo de tempo definido, estando os trabalhos identificados entre os anos de 2008 e 2011, totalizando 21 trabalhos. Porém, destes 21 estudos, os que tratam de RNFs na modelagem de processos são apenas 6. Estes 6 trabalhos foram discutidos com mais detalhe pelos autores.

Na nossa revisão sistemática, optamos por não definir um intervalo de tempo na busca de estudos com o propósito de alcançar mais resultados. Por isso, não utilizamos os recursos das bases de dados que permitem ao pesquisador delimitar um intervalo de tempo em que os trabalhos serão buscados. Ao todo, em nossa pesquisa, foram identificados 13 estudos que tratam de RNFs na modelagem de processos de negócio. Além de descrevermos cada um dos estudos, também fizemos um mapeamento entre o tipo de 
representação de RNF no modelo. Ao todo foram identificados 6 tipos de representações diferentes.

Este mapeamento pode ajudar pesquisadores na identificação de um grupo de trabalhos que representam RNF de uma determinada maneira, permitindo um estudo mais criterioso desse grupo, identificando pontos fortes e fracos dos trabalhos e fazendo comparações entre os mesmos. Também pode-ser fazer comparações entre os tipos de representações e propor melhorias para as abordagens analisadas.

\section{Ameaças à Validade}

Em consequência à restrição imposta pela ferramenta $\mathrm{StArt}^{2}$ utilizada no processo desta revisão sistemática, o critério de seleção de bases de dados em que as mesmas devem permitir exportar o bibtex de todos os estudos retornados na busca foi incluído. Portanto, bases relevantes como ACM, dentre outras, não foram consideradas.

Também não poderíamos usar nossas strings de busca na biblioteca Springerlink, o que pode levar a uma redução considerável de estudos. Para atenuar esta ameaça, utilizamos a SCOPUS, que é uma base de dados grande que inclui artigos da springer. A classificação dos estudos foram baseados em nosso julgamento, contudo, pode ocorrer que alguns estudos tenham sido classificados incorretamente. Dado as limitações das engines de busca, não temos como garantir que todos os estudos relevantes publicados estão inseridos nesta revisão sistemática. Além disso, não consideramos artigos industriais, dissertação, tese de doutorado, livros ou resultados não publicados.

\section{Conclusão e Trabalhos Futuros}

Requisitos não-funcionais são atributos difíceis de serem capturados e representados, assim como informações de contexto. A dificuldade de lidar com RNFs não está presente somente na modelagem de processos, mas também em outras áreas como em linha de produtos de software (LPS). [Pereira and Teixeira Filho 2011] fizeram um mapeamento entre ferramentas e funcionalidades de LPS, e das 22 ferramentas analisadas, apenas 2 identificam features através de RNF.

Informações de contexto são essenciais em processos de negócio e sistemas de software para alcançar flexibilidade. Por isso, a modelagem explícita de contexto é especialmente útil na modelagem de processos de negócio onde o significado de qualquer elemento do modelo deverá ser definido precisamente [Balabko and Wegmann 2003]. Por causa da grande dificuldade em encontrar abordagens que representam RNFs e contexto, foi realizado uma revisão sistemática de literatura, onde foi possível identificar trabalhos que tratam de RNF e contexto em modelos de processos de negócio.

As empresas e acadêmicos que pretendem modelar processos levando em consideração os RNFs e contexto, devem utilizar o quanto antes a análise dos trabalhos realizada nesta pesquisa, pois, estarão consequentemente identificando qual abordagem melhor se encaixa no ambiente em que será trabalhado, reduzindo o tempo de busca de uma abordagem e evitando erros, escolhendo uma abordagem que não se encaixa nos requisitos da modelagem. Classificações foram realizadas com o intuito de agrupar os trabalhos em relação a diferentes fatores como, por exemplo, as linguagens de modelagem de processos utilizadas para representar RNFs, os tipos de representações de RNFs,

\footnotetext{
${ }^{2}$ http://lapes.dc.ufscar.br/software/start-tool
} 
os métodos utilizados para representar contexto, assim como a técnica de captura de contexto utilizada, dentre outros.

Como trabalhos futuros, pretendemos realizar uma análise mais crítica dos resultados alcançados. Buscando fazer comparações entre abordagens, identificando aspectos positivos, negativos e oportunidades de melhorias. O estudo de [Nurcan 2008] servirá de ponto de partida para esta análise mais criteriosa, já que possui uma lista de requisitos importantes que podem ser usados para comparar abordagens que tratam de contexto em processos de negócio.

\section{Agradecimentos}

Este trabalho foi financiado pela FACEPE (Fundação de Amparo à Ciência e Tecnologia do Estado de Pernambuco), processo PBPG-0506-1.03/11.

\section{Referências}

Aburub, F., Odeh, M., and Beeson, I. (2007). Modelling non-functional requirements of business processes. Information and Software Technology, 49(11):1162-1171.

Balabko, P. and Wegmann, A. (2003). Context based reasoning in business process models. In Information Reuse and Integration, 2003. IRI 2003. IEEE International Conference on, pages 120-128. IEEE.

Bartolini, C., Bertolino, A., De Angelis, G., Ciancone, A., and Mirandola, R. (2012). Non-functional analysis of service choreographies. In Principles of Engineering Service Oriented Systems (PESOS), 2012 ICSE Workshop on, pages 8-14. IEEE.

Bocciarelli, P. and D'Ambrogio, A. (2011). A bpmn extension for modeling non functional properties of business processes. In Proceedings of the 2011 Symposium on Theory of Modeling \& Simulation: DEVS Integrative M\&S Symposium, pages 160-168. Society for Computer Simulation International.

Cardoso, E., Almeida, J., Guizzardi, G., and Guizzardi, R. (2009). Eliciting goals for business process models with non-functional requirements catalogues. Enterprise, Business-Process and Information Systems Modeling, pages 33-45.

Chung, L., Brian, A., Eric, Y., and John, M. (2000). Non-functional requirements in software engineering. Non-Functional Requirements in Software Engineering.

Dyba, T., Kitchenham, B., and Jorgensen, M. (2005). Evidence-based software engineering for practitioners. Software, IEEE, 22(1):58-65.

J. Biolchini, P. G. Mian, A. C. N. e. G. H. T. (2005). Systematic review in software engineering: relevance and utility. Technical report, COPPE/UFRJ, Rio de Janeiro.

J. H. Little, J. C. and Pillai, V. (2008). Systematic Review and Meta-Analysis. USA: Oxford University Press, 1 edition.

Kedad, Z. and Loucopoulos, P. (2011). Considering quality factors for business processes during requirement engineering. In Research Challenges in Information Science (RCIS), 2011 Fifth International Conference on, pages 1-9. IEEE.

Khaluf, L., Gerth, C., and Engels, G. (2011). Pattern-based modeling and formalizing of business process quality constraints. In Advanced Information Systems Engineering, pages 521-535. Springer. 
Lapouchnian, A., Yu, Y., and Mylopoulos, J. (2007). Requirements-driven design and configuration management of business processes. Business Process Management, pages 246-261.

Mafra, S. and Travassos, G. (2006). Estudos primários e secundários apoiando a busca por evidência em engenharia de software. Rio de Janeiro: Programa de Engenharia de Sistemas e Computação. RT-ES, 687(06).

Mian, P., Conte, T., Natali, A., de Almeida Biolchini, J., Mendes, E., and Travassos, G. (2005). Lessons learned on applying systematic reviews to software engineering. In the Workshop Series On Empirical Software Engineering (Wsese).

Nurcan, S. (2008). A survey on the flexibility requirements related to business processes and modeling artifacts. In Hawaii International Conference on System Sciences, Proceedings of the 41st Annual, pages 378-378. IEEE.

Pavlovski, C. and Zou, J. (2008). Non-functional requirements in business process modeling. In Proceedings of the fifth Asia-Pacific conference on Conceptual ModellingVolume 79, pages 103-112. Australian Computer Society, Inc.

Pereira, T. and Teixeira Filho, G. (2011). Software product lines: An analysis of tools that support the application requirements engineering's phases. 9th CONTECSI International Conference on Information Systems and Technology Management.

Saeedi, K., Zhao, L., and Sampaio, P. (2010). Extending bpmn for supporting customerfacing service quality requirements. In Web Services (ICWS), 2010 IEEE International Conference on, pages 616-623. IEEE.

Salles, G. and Fantinato, M. (2012). Análise da incorporação de requisitos não funcionais na modelagem de processos de negócio. VIII Simposio Brasileiro de Sistemas de Informacaoo (SBSI 2012) Trilhas Tecnicas.

Sampaio, R. and Mancini, M. (2007). Estudos de revisão sistemática: um guia para síntese criteriosa da evidência científica; systematic review studies: a guide for careful synthesis of the scientific evidence. Rev. bras. fisioter, 11(1):83-89.

Santos, E., Pimentel, J., Castro, J., and Finkelstein, A. (2012). On the dynamic configuration of business process models. Enterprise, Business-Process and Information Systems Modeling, pages 331-346.

Serrano, N., Caballero, I., and García, F. (2009). Extending bpmn to support the modeling of data quality issues. In Int. Conf. on Inform. Quality (ICIQ), pages 46-60.

Wolter, C. and Meinel, C. (2010). An approach to capture authorisation requirements in business processes. Requirements engineering, 15(4):359-373.

Xavier, L., Alencar, F., Castro, J., and Pimentel, J. (2009). Integração de requisitos nãofuncionais a processos de negócio: Integrando bpmn e nfr. MSC, Universidade Federal de Pernambuco.

Xia, Y. and Wei, J. (2008). Context-driven business process adaptation for ad hoc changes. In e-Business Engineering, 2008. ICEBE'08. IEEE International Conference on, pages 53-60. IEEE. 